Review

\title{
Open and Dense Topological Transitivity of Extensions by Non-Compact Fiber of Hyperbolic Systems: A Review
}

\author{
Viorel Nitica $^{1,2, *}$ and Andrei Török ${ }^{2,3}$ \\ ${ }^{1}$ Department of Mathematics, West Chester University, West Chester, PA 19383, USA \\ ${ }^{2}$ Institute of Mathematics of the Romanian Academy, P.O. Box 1-764, RO-70700 Bucharest, Romania \\ ${ }^{3}$ Department of Mathematics, University of Houston, 651 PGH, Houston, TX 77204-3008, USA; \\ E-Mail: torok@math.uh.edu
}

* Author to whom correspondence should be addressed; E-Mail: vnitica@wcupa.edu;

Tel.: +610-430-4411.

Academic Editor: Sidney A. Morris

Received: 12 December 2014 / Accepted: 26 January 2015 / Published: 4 February 2015

\begin{abstract}
Currently, there is great renewed interest in proving the topological transitivity of various classes of continuous dynamical systems. Even though this is one of the most basic dynamical properties that can be investigated, the tools used by various authors are quite diverse and are strongly related to the class of dynamical systems under consideration. The goal of this review article is to present the state of the art for the class of Hölder extensions of hyperbolic systems with non-compact connected Lie group fiber. The hyperbolic systems we consider are mostly discrete time. In particular, we address the stability and genericity of topological transitivity in large classes of such transformations. The paper lists several open problems and conjectures and tries to place this topic of research in the general context of hyperbolic and topological dynamics.
\end{abstract}

Keywords: stable topological transitivity; hyperbolic system; Anosov diffeomorphism; extension with Lie group fiber 


\section{Introduction}

A dynamical system is a continuous map $f$ of a topological space $X$. We emphasize that in this paper, $X$ will be mostly a non-compact set and $f$ will be invertible. Given a dynamical system $(X, f)$, a basic property that one may study is topological transitivity, that is the existence of a dense forward orbit $x, f(x), f^{2}(x), \ldots$ If $X$ is locally compact separable without isolated points, then $(X, f)$ is topologically transitive if and only if for any non-empty open subsets $U, V \subset X$, there exists $n \geq 1$, such that $f^{n}(U) \cap V \neq \emptyset$. The topological spaces that we will work with, Riemannian manifolds and phase spaces of shifts of the finite type, satisfy these conditions. Let us observe that other, sometimes equivalent with ours, definitions are introduced in the literature for topological transitivity. One may refer to the survey papers of Blanchard [1] or Kolyada-Snoha [2] for a more in-depth discussion and other definitions/interpretations of topological transitivity. This notion is also closely related to the notion of topological chaos introduced by Devaney [3]. The original definition of topological chaos given by Devaney, in addition to topological transitivity, requires the existence of a dense set of periodic points and the sensitive dependence of initial data for the dynamical system. It was later shown by Banks et al. [4] that the sensitivity of the initial data is a consequence of the other two conditions.

Various examples of topological transitive transformations are constructed in the literature. In some respects, topological transitivity is the topological counterpart of ergodicity. We recall that a probability measure $\mu$ on a measurable space $X$ is ergodic with respect to a measurable map $f: X \rightarrow X$ if and only if the only $f$-invariant subsets, up to subsets of measure zero, are $X$ and the empty set. If $X$ is compact topological space and the continuous map $f$ has an invariant Borel probability measure $\mu$, which is positive on open sets, then topological transitivity is implied by ergodicity. It was shown by Oxtoby and Ulam [5] that ergodicity is a residual property, in the set of homeomorphisms of a manifold $X$ of dimension at least two, if $\mu$ is a nonatomic measure of full support with $\mu(\partial X)=0$. We recall that a residual property for a complete metric space is one that is valid for a second Baire category subset.

Examples of topological transitive transformations of the plane are constructed by Besicovitch [6,7] and Shnirelman [8]. Their work was generalized by Sidorov [9], who constructed topological transitive extensions of a topologically transitive map with fiber an arbitrary Banach space.

The class of hyperbolic dynamical systems, introduced in the 1960s-1970s by Anosov and Sinai [10] in the USSR and by Bowen [11] and Smale [12] in the USA, provides many examples of ergodic and, in particular, topologically transitive transformations. Hyperbolic systems have a splitting of the tangent bundle into two invariant subbundles, one contracting and one expanding. These bundles are integrable into stable/unstable foliations. A standard reference for the theory of hyperbolic dynamical systems is the monograph of Katok and Hasselblatt [13]. During our exposition, we will assume as known or already defined many standard notions discussed there. Similar techniques can be applied to continuous dynamical systems, such as hyperbolic flows. Building on an argument of Hopf [14], who proved the ergodicity of the geodesic flow of a surface of negative curvature, Anosov [15] proved the ergodicity of the geodesic flow of any manifold of negative curvature. A key ingredient of the proof is the existence of invariant stable and unstable foliations for the geodesic flow. These foliations are, in general, only transversally Hölder, but exhibit the absolute continuity of the holonomy maps; this allows the Hopf argument to be carried out. 
Partially hyperbolic diffeomorphisms were introduced in the 1980s by Brin and Pesin [16] as a generalization of hyperbolic diffeomorphisms. Partially hyperbolic diffeomorphisms have a splitting of the tangent bundle into three invariant subbundles, one contracting, one expanding and one, called the center bundle, for which the expansion/contraction is in-between. The contracting and expanding subbundles are always integrable into stable/unstable foliations. It was expected that, in the presence of a smooth invariant volume, ergodicity with respect to the volume measure should be true for many of these transformations. Brin and Pesin [16] proved ergodicity under the assumptions that the stable/unstable foliations are smooth and form an accessible pair, that is, one can travel from any point in the manifold to any other point in the manifold via a path built out of segments sitting inside stable/unstable leaves. The argument was carried forward by Grayson, Pugh and Shub [17], Pugh and Shub [18] and Burns and Wilkinson [19]. The state of the art in this direction is that the accessibility of the pair of stable/unstable foliations implies ergodicity. Nevertheless, accessibility turned out to be difficult to prove. Some progress was done in the case of a one-dimensional center subbundle; see, e.g., [20]. Hertz [21] proved the stable ergodicity of certain linear automorphisms of the torus. Furthermore, Dolgopyat and Wilkinson [22] proved that stable accessibility is dense in the $C^{1}$-topology for the class of volume preserving partially hyperbolic diffeomorphisms. Pugh and Shub conjectured that in $C^{2}$ and even $C^{r}, r \geq 2$ topology, accessibility is open and dense.

If the invariant volume does not exist, then even in the presence of partial hyperbolicity, different tools are needed in order to study topological transitivity. Given a dynamical system $(X, f)$, a point $x \in X$ is called recurrent if for any neighborhood $U$ of $x$, there exists a positive integer $n \geq 1$, such that $f^{n}(x) \in U$. In particular, any periodic point is recurrent. Brin [23] proved that a $C^{1}$ diffeomorphism that has an accessible pair of stable/unstable foliations and a dense set of recurrent points is topologically transitive. This result has, nevertheless, limited applicability due to the difficulty of proving the accessibility and density of recurrent points. In particular, it is difficult to exhibit a dense set of periodic points for such transformations. Open sets of transitive partially hyperbolic diffeomorphisms are found by Bonatti and Diaz [24], building on previous examples found by Shub [25].

A robust obstruction to topological transitivity is the existence of a trapping region, i.e., a non-empty open proper subset $U \subset M$, such that $f(\bar{U}) \subseteq U$. When this obstruction does not occur, it follows from the work of Bonatti and Crovisier [26] that a generic $C^{1}$ diffeomorphism of a compact Riemannian manifold is topologically transitive. This result relies on the Pugh-Hayashi [27-29] closing lemma, and it is not available beyond the $C^{1}$ category.

Another direction currently pursued in the literature is that of linear topological chaos, that is, the study of topological chaos for infinite dimensional continuous linear operators [30]. The techniques employed in linear topological chaos are quite different from those employed in the study of hyperbolic dynamical systems and will not be discussed in this review.

In the rest of this review paper, we summarize the results about the topological transitivity for various classes of non-compact Lie group extensions of hyperbolic systems. These classes of dynamical systems can be thought of as thin classes of partially hyperbolic systems. 


\section{Lie Group Extensions of Hyperbolic Systems: Basic Definitions and the Main Conjecture}

Definition 1. Consider a continuous transformation $f: X \rightarrow X$, a Lie group $\Gamma$ and a continuous map $\beta: X \rightarrow \Gamma$ called a cocycle. These determine a skew product, or $\Gamma$-extension,

$$
f_{\beta}: X \times \Gamma \rightarrow X \times \Gamma, \quad f_{\beta}(x, \gamma)=(f x, \gamma \beta(x))
$$

It is assumed throughout the rest of the paper that $X$ is a hyperbolic basic set. Of interest to us is whether non-compact Lie group extensions of a hyperbolic basic set are typically topologically transitive.

Definition 2. Let $\left(M, d_{M}\right)$ be a smooth manifold endowed with a Riemannian metric. Let $f: M \rightarrow M$ be a smooth diffeomorphism and $X \subset M$ a compact and $f$-invariant subset of $M$. We say that $X$ is hyperbolic if there exists a continuous $D$ f-invariant splitting $E^{s} \oplus E^{u}$ of the tangent bundle $T_{X} M$ and constants $C_{1}>0,0<\lambda<1$, such that for all $n \geq 0$ and $x \in X$, we have:

$$
\begin{gathered}
\left\|\left(D f^{n}\right)_{x} v\right\| \leq C_{1} \lambda^{n}\|v\|, v \in E_{x}^{s} \\
\left\|\left(D f^{-n}\right)_{x} v\right\| \leq C_{1} \lambda^{n}\|v\|, v \in E_{x}^{u}
\end{gathered}
$$

If $X$ coincides with $M$, then $f$ is called hyperbolic, or Anosov, diffeomorphism.

We say that $X$ is locally maximal if there exists an open neighborhood $U$ of $X$, such that every compact $f$-invariant set of $U$ is contained in $X$. A locally maximal hyperbolic set $X$ is a basic set for $f: M \rightarrow M$ if $f: X \rightarrow X$ is transitive and $X$ does not consist of a single periodic orbit.

We present some motivation for the study of Lie group extensions of hyperbolic systems. These transformations have many common properties with partially hyperbolic diffeomorphisms. If the fiber is a compact connected Lie group, the cocycle $\beta$ is at least $C^{1}$ and the hyperbolic basic set $(X, f)$ is an Anosov diffeomorphism, then the extension is a partially hyperbolic diffeomorphism. In general, we will see that if a certain bunching condition for the center direction holds, then $f_{\beta}$ has stable and unstable foliations. If the Lie group $\Gamma$ is not compact, the extension $f_{\beta}$ acts on a space that does not support an invariant probability measure. Thus, the class of non-compact Lie group extensions can be considered as a test bed for the more general class of partially hyperbolic diffeomorphisms that do not have a nice invariant probability measure.

Given a connected Lie group $\Gamma$ and a cocycle $\beta: X \rightarrow \Gamma$, we consider the $\Gamma$-extension $f_{\beta}: X \times \Gamma \rightarrow$ $X \times \Gamma$. We say that the cocycle $\beta$ is topologically transitive (for brevity, transitive,) if the corresponding skew product $f_{\beta}$ is transitive.

Let $L \Gamma$ be the Lie algebra of $\Gamma$. We denote by $e_{\Gamma}$ the identity element of $\Gamma$. Let Ad denote the adjoint representation of $\Gamma$ on $L \Gamma$. Let $\|\cdot\|$ be a norm on $L \Gamma$. There is a metric $d$ on $\Gamma$ with the following properties (see Pollicott and Walkden [31]):

1. $d\left(\gamma \gamma_{1}, \gamma \gamma_{2}\right)=d\left(\gamma_{1}, \gamma_{2}\right)$

2. $d\left(\gamma_{1} \gamma, \gamma_{2} \gamma\right) \leq\|\operatorname{Ad}(\gamma)\| d\left(\gamma_{1}, \gamma_{2}\right)$;

for any $\gamma, \gamma_{1}, \gamma_{2} \in \Gamma$. 
Definition 3. Let $f: X \rightarrow X$ be a map and $\beta: X \rightarrow \Gamma$ a cocycle. For $k \geq 1$, we write $f_{\beta}^{k}(x, \gamma)=\left(f^{k} x, \gamma \beta(k, x)\right)$, where:

$$
\beta(k, x)=\beta(x) \beta(f x) \cdots \beta\left(f^{k-1} x\right)=\prod_{j=0}^{k-1} \beta\left(f^{j} x\right)
$$

(occasionally, we use the last formula to keep notation simple; its meaning is the ordered product given by the middle expression).

If $Q$ is a trajectory of $f$ of length $k$ (i.e., $Q=\left\{x, f(x), \ldots, f^{k-1}(x)\right\}$ for some $x$ ), then we define the height of $\beta$ over $Q$ to be $\beta(Q)=\beta(k, x)$. In particular, if $x$ is a periodic point of period $\ell$, then the height of the corresponding periodic orbit $P$ is $\beta(P)=\beta(\ell, x)$.

By abuse of notation, we often refer to "the periodic orbit $P$ " instead of "the orbit of the periodic point $x "$ when $x$ is clear from the context.

Definition 4. Given a cocycle $\beta: X \rightarrow \Gamma$ over $f: X \rightarrow X$, define $\mu \geq 1$ to be:

$$
\mu=\max \left\{\lim _{n \rightarrow \infty} \sup _{x \in X}\|A d(\beta(n, x))\|^{1 / n}, \lim _{n \rightarrow \infty} \sup _{x \in X}\left\|A d(\beta(n, x))^{-1}\right\|^{1 / n}\right\}
$$

For $f$ fixed, we say that the cocycle $\beta$ has subexponential growth if $\mu=1$.

Remark 1. The subexponential growth condition is automatically satisfied for any cocycle if the group $\Gamma$ is compact, nilpotent or a semidirect product of compact and nilpotent. This follows from the well-known result that nilpotent Lie groups have polynomial growth [32].

Recall the definition of cohomology:

Definition 5. Let $\Gamma$ be a topological group. If $\beta_{1}, \beta_{2}: X \rightarrow \Gamma$ are continuous functions and $f: X \rightarrow X$ is a continuous map, then $\beta_{1}, \beta_{1}$ are called cohomologous (over $f$ ) if there exists a continuous map $u: X \rightarrow \Gamma$, such that:

$$
\beta_{1}=(u \circ f) \beta_{2} u^{-1}
$$

In [33], we proposed a general conjecture about topological transitivity in the class of Hölder cocycles. We start by observing that if the cocycle $\beta$ takes values in a proper closed sub-semigroup $S$ of the fiber $\Gamma$, then obviously $f_{\beta}$ is not transitive. An example is the group $\Gamma=\mathbb{R}$ with sub-semigroup $S$ consisting of the set of non-negative numbers. As $\operatorname{Int} S \neq \emptyset$, we can construct open sets of nontransitive $\mathbb{R}$-extensions. Another example is the group $\Gamma=S L(n, \mathbb{R})$ with sub-semigroup $S$ consisting of matrices with non-negative entries. Since $\operatorname{Int} S \neq \emptyset$, again we can construct open sets of nontransitive $S L(n, \mathbb{R})$-extensions.

Our conjecture is that this situation is the only essential obstruction to transitivity.

Conjecture 1 (Main Conjecture). Assume that $X$ is a hyperbolic basic set for $f: X \rightarrow X$ and $\Gamma$ is a finite-dimensional connected Lie group. Among the Hölder cocycles $\beta: X \rightarrow \Gamma$ with subexponential growth that are not cohomologous to a cocycle with values in a maximal sub-semigroup of $\Gamma$ with a non-empty interior, there is a Hölder open and dense set for which the extension $f_{\beta}$ is transitive. 


\section{Statements of Available Results}

The conjecture is proven for various classes of Lie groups. The techniques used so far are quite diverse and seem to depend heavily on the particular properties of the group that appears in the fiber.

\section{1. Г Compact Connected Lie Group}

We start by observing that in this case, closed sub-semigroups coincide with closed subgroups and that there are no proper sub-semigroups with a nonempty interior. It was proven by Brin [23] that if the fiber $\Gamma$ is a compact connected Lie group, then topologically transitive extensions of a transitive Anosov diffeomorphism contain a set that is open and dense in the $C^{2}$-topology. An extension of Brin's general transitivity result is obtained in [34,35], in which accessibility is replaced by $\epsilon$-accessibility for any $\epsilon>0$. This improvement allows one to consider extensions with a disconnected base, such as subshifts of the finite type.

As observed in [20], Brin's result also holds in the Hölder topology. In fact, over an Anosov diffeomorphism, for any $r>0$, the $C^{r}$ cocycles that are transitive contain a Hölder-open (meaning $C^{s}$-open for any $\left.s \in(0,1), s \leq r\right)$ and $C^{r}$-dense set, and this result generalizes to extensions of a hyperbolic attractor. The latter result does not hold for extensions of general hyperbolic basic sets when $r<1$ (in particular, the result is false if $(X, f)$ is topologically conjugate to a subshift of finite type and $\Gamma$ is a torus: the interior of the transitive $C^{r}$-cocycles contains no cocycle of higher smoothness). However, for compact group extensions of general hyperbolic basic sets, Field et al. [36] proved that the transitive extensions contain a set that is: (i) Hölder open and dense (proving the Main Conjecture 1); and (ii) $C^{2}$-open, $C^{r}$-dense for all $r \geq 2$. See, also, [37-39].

Burns and Wilkinson [40] generalized Brin's result by showing the stability of the ergodicity of the extensions with compact fiber for perturbations in the class of $C^{r}$ diffeomorphisms.

\section{2. $\Gamma=\mathbb{R}^{n}$}

In this case, the maximal sub-semigroups with non-empty interior are the half-spaces whose bounding hyperplane contains the origin. Hence, stable transitivity is certainly not a generic property of $\mathbb{R}^{n}$-extensions. However, there are no further obstructions. We recall that a continuous map $f$ of a topological space $X$ is called weakly topologically mixing if $f \times f$ is topologically transitive. We can associate with each periodic orbit $f^{n} x=x$ a height $\beta(n, x):=\sum_{i=0}^{n-1} \beta\left(f^{i} x\right)$. We denote by $\mathrm{PD}_{\beta}=\left\{\beta(n, x): f^{n} x=x, x \in X\right\}$ the collection of all heights over the closed orbits of $f$.

Nitica and Pollicott [41] proved the following result:

Theorem 1. Let $X$ be an infranilmanifold, $f: X \rightarrow X$ an Anosov diffeomorphism and $\beta: X \rightarrow \mathbb{R}^{n} a$ Hölder cocycle. Then, the following are equivalent:

1. the cocycle $\beta$ is not cohomologous to a cocycle that takes values in a half-space;

2. the set $P D_{\beta}$ is not separated by any hyperplane passing through the origin;

3. the extension $f_{\beta}$ is transitive;

4. the extension $f_{\beta}$ is $C^{0}$-stably transitive; 
5. the extension $f_{\beta}$ is weakly mixing;

6. the extension $f_{\beta}$ is $C^{0}$-stably weakly mixing;

7. for any direction in $\mathbb{R}^{n}$, there exist orbits of $f_{\beta}$ that are unbounded in the positive sense and orbits that are unbounded in the negative sense (i.e., $\forall v \in \mathbb{R}^{n}-\{0\}, \exists x, y \in X, \forall N>0, \exists n, m \geq 0$ such that $\langle\beta(n, x), v\rangle \geq N$ and $\langle\beta(m, y), v\rangle \leq-N)$.

Remark 2. We note that, due to a result of Bousch [42], one can check if a cocycle $\beta$ is cohomologous to one that takes values in a half-space by looking at the periodic data $P D_{\beta}$.

Therefore, one has a complete dichotomy for an $\mathbb{R}^{n}$-extension $f_{\beta}$ over an infranil Anosov diffeomorphism: either it is transitive (and, hence, stably transitive), or $\beta$ is cohomologous to a cocycle with values in such a half-space. Moreover, the transitive Hölder $\mathbb{R}^{n}$-extensions $f_{\beta}$ are actually $C^{0}$-stably transitive, that is, if $\beta^{\prime}$ is $C^{0}$-close enough to $\beta$ and $f_{\beta}$ is transitive, then $f_{\beta^{\prime}}$ is transitive.

A crucial ingredient in the proof of Theorem 1 is that the induced map in the first Čech cohomology groups $f^{*}: H^{1}(X, \mathbb{Z}) \rightarrow H^{1}(X, \mathbb{Z})$ does not have one as an eigenvalue. This is known to be the case for an Anosov diffeomorphism of an infranilmanifold, and it is an important open question as to whether this is the case for all Anosov diffeomorphisms ( $c f$. [43]) (indeed, it is an open question as to whether there are Anosov diffeomorphisms on spaces other than infranilmanifolds).

Moss and Walkden [44] replace the condition about the induced map in cohomology by the weaker condition that the first Čech cohomology group $H^{1}(X, \mathbb{Z})$ has finite rank, where $X$ is a hyperbolic basic set. Moreover, the action in the base is extended to hyperbolic flows. Their proof gives an explicit and global description of the set of functions $\beta$ that give rise to transitive skew-products in terms of the cohomology of the hyperbolic basic set $X$.

We recall that a continuous map $f$ of a topological space $X$ is called topologically mixing if for any open subsets $U, V \subseteq X$, there exists a positive integer $N$, such that $f^{n}(U) \cap V \neq \emptyset$ for any $n \geq N$. The following problem seems to be open.

Problem 1. Find a topologically mixing $\mathbb{R}$-extension of an Anosov diffeomorphism.

For general hyperbolic basic sets, transitive $\mathbb{R}^{n}$-extensions need not be stably transitive. However, let $S$ denote the set of cocycles that are not cohomologous to a cocycle with values in a half-space. For cocycles in $S$, Field et al. [36] proved a result identical to that stated above for compact group extensions. Again this proves the Main Conjecture 1 for $\mathbb{R}^{n}$-extensions. Similar results hold for general Abelian finite-dimensional Lie groups $\Gamma=\mathbb{R}^{n} \times \mathbb{T}^{d}$, where $\mathbb{T}^{d}$ is a $d$-dimensional torus.

\section{3. $\Gamma$ Is a Euclidean-Type Group}

An important test case of a Euclidean-type group is the special Euclidean group, which is the semidirect product $\Gamma=S E(n)=S O(n) \ltimes \mathbb{R}^{n}$ with the action of $S O(n)$ on $\mathbb{R}^{n}$ given by the usual matrix multiplication. In this case, it is easy to see that there are no proper sub-semigroups with non-empty interior. It is shown in $[33,45,46]$ that when $n$ is even, the set of cocycles that are transitive is Hölder-open and $C^{r}$-dense, thus solving the conjecture in this case. The conjecture remains open for $n \geq 3$ odd. The difference between the case $n$ odd and $n$ even is due to the different behavior of a generic element in 
$\Gamma$ : if $n$ is even, then for a residual set of elements in $\Gamma$, the closure of the semigroup generated by an element is a compact subgroup of $\Gamma$; if $n$ is odd, then for a residual set of elements in $\Gamma$, the closure of the semigroup generated by an element is an unbounded subset of $\Gamma$.

Problem 2. For $0<\alpha<1$, find a $C^{\alpha}$-stable transitive $S E(3)$-extension of an Anosov diffeomorphism.

In this direction, we show in [47] that for $S E(n)$-extensions, $n \geq 3$ odd, the transitivity is generic.

Theorem 2. Let $X$ be a basic hyperbolic set for $f: X \rightarrow X$. Let $r>0$, and let $n \geq 3$ be odd. Amongst the $C^{r}$ cocycles $\beta: X \rightarrow S E(n)$, the transitive cocycles form a residual set.

More generally, one may consider Euclidean-type groups of the form $\Gamma=G \ltimes \mathbb{R}^{n}$, where $G$ is a compact connected Lie group acting linearly (and orthogonally) on $\mathbb{R}^{n}$, and the group multiplication is given by:

$$
\left(g_{1}, v_{1}\right)\left(g_{2}, v_{2}\right)=\left(g_{1} g_{2}, v_{1}+g_{1} v_{2}\right)
$$

Let Fix $G=\left\{v \in \mathbb{R}^{n}: g v=v\right.$ for all $\left.g \in G\right\}$. Set $\pi: \Gamma \rightarrow$ Fix $G$ to be the projection onto the $\mathbb{R}^{n}$-component and then orthogonal projection onto Fix $G$. If Fix $G \neq\{0\}$, then there is an obvious obstruction to transitivity, namely that $\pi \beta: X \rightarrow$ Fix $G$ takes values in a half-space. More generally, if $\pi \beta$ is cohomologous to a cocycle with values in a half space, then $f_{\beta}$ is not transitive. This is the only obstruction in generalizing Theorem 2 to general Euclidean-type groups.

Theorem 3. Let $X$ be a basic hyperbolic set for $f: X \rightarrow X$, and let $\Gamma=G \ltimes \mathbb{R}^{n}$ be a Euclidean-type group. Let $r>0$. Define $\mathcal{S}$ to be the space of $C^{r}$ cocycles $\beta: X \rightarrow \Gamma$ for which $\pi \beta: X \rightarrow$ Fix $G$ is not cohomologous to a cocycle with values in a half-space.

Then, $\mathcal{S}$ is an open subset of the space of $C^{r}$ cocycles, and the transitive cocycles $\beta: X \rightarrow \Gamma$ form a residual subset of $\mathcal{S}$.

Remark 3. (1) If Fix $G=0$, then there is no obstruction to transitivity, so Theorem 2 is a special case of Theorem 3.

(2) By a standard argument, the set of transitive $C^{r}$ cocycles can be written as a countable intersection of $C^{r}$-open sets. We include the argument below. Hence, it suffices to prove the density in Theorems 2 and 3.

Choose a countable basis $\left\{U_{k}\right\}_{k}$ of the topology on $X \times \Gamma$ and denote by $C_{k, \ell}^{r}$ the $C^{r}$ cocycles $\beta \in \mathcal{S}$ for which there is a positive integer $n$, such that $f_{\beta}^{n}\left(U_{k}\right) \cap U_{\ell} \neq \emptyset$. Each set $C_{k, \ell}^{r}$ is clearly $C^{r}$-open, and $f$ is transitive if and only if $\beta$ is in each of the sets $C_{k, \ell}^{r}$.

\section{4. Г Is a Nilpotent Lie Group}

Definition 6. For $n \geq 1$, let $\mathcal{H}_{n}$ denote the group consisting of matrices of the form:

$$
(a, b, c):=\left(\begin{array}{ccc}
1 & a^{T} & c \\
0 & I_{n} & b \\
0 & 0 & 1
\end{array}\right) \in \operatorname{Mat}_{n+2}(\mathbb{R})
$$


where $a, b \in \mathbb{R}^{n}, c \in \mathbb{R}$ and $I_{n}$ is the $n$-dimensional identity matrix.

Remark 4. (1) We can identify $\mathcal{H}_{n}$ with $\mathbb{R}^{n} \oplus \mathbb{R}^{n} \oplus \mathbb{R}$ endowed with the multiplication:

$$
(a, b, c)(A, B, C)=\left(a+A, b+B, c+C+a^{T} B\right)
$$

where $a, b, A, B \in \mathbb{R}^{n}, c, C \in \mathbb{R}$

(2) $\mathcal{H}_{1}$ is the standard three-dimensional Heisenberg group.

The center of $\mathcal{H}_{n}$ is $\left[\mathcal{H}_{n}, \mathcal{H}_{n}\right]=\{(0,0, c)\}=\mathbb{R}$. Denote $\widehat{\mathcal{H}}_{n}=\mathcal{H}_{n} / \mathbb{R} \cong \mathbb{R}^{2 n}$. If $\beta: X \rightarrow \mathcal{H}_{n}$ is a cocycle, denote by $\widehat{\beta}: X \rightarrow \widehat{\mathcal{H}}_{n}$ the corresponding quotient cocycle. There is an obvious obstruction to transitivity, namely that $\widehat{\beta}: X \rightarrow \widehat{\mathcal{H}}_{n} \cong \mathbb{R}^{2 n}$ takes values in a half-space bounded by a hyperplane passing through the origin (for brevity, call this a half-space from now on). More generally, if $\widehat{\beta}$ is cohomologous to a cocycle with values in a half-space, then $f_{\beta}$ is not transitive.

If $r>0$, let $\mathcal{S}^{r}\left(X, \mathcal{H}_{n}\right)$ be the set of $C^{r}$ cocycles $\beta: X \rightarrow \mathcal{H}_{n}$ for which $\widehat{\beta}$ is not cohomologous to a cocycle with values in a half-space. The main result in [48], improving on a weaker result showing only genericity and proven in [49], is:

Theorem 4 ([48, Theorem 1.4]). Assume that $X$ is a hyperbolic basic set for $f: X \rightarrow X$. Let $r>0$. Then $\mathcal{S}^{r}\left(X, \mathcal{H}_{n}\right)$ contains a dense and open set of transitive cocycles.

More precisely, we prove:

Theorem 5 ([48, Theorem 1.5]). Let $X$ be a hyperbolic basic set for $f: X \rightarrow X$ and $\beta: X \rightarrow \mathcal{H}_{n} a$ Hölder cocycle. If $\hat{\beta}: X \rightarrow \mathbb{R}^{2 n}$ is transitive, then so is $\beta$.

This implies Theorem 4, because, by [36,41]:

Theorem 6 ([48, Theorem 1.6]). For $r>0$, there is an open and dense set in $\mathcal{S}^{r}\left(X, \mathbb{R}^{d}\right)$ consisting of transitive cocycles.

A new technical tool needed in the proof of Theorem 4, which is of independent interest, is a diophantine approximation result, which shows the existence of an infinite set of approximate positive integer solutions for a diophantine system of equations consisting of a quadratic indefinite form and several linear equations, provided exact solutions exist over $\mathbb{R}$. The set of approximate solutions can be chosen to point in a certain direction; this direction can be chosen from a residual subset of full measure of the set of real directions solving exactly the system of equations.

Theorem 7 ([48, Theorem 6.2]). For $d \geq 2$, assume given in $\mathbb{R}^{d}$ a (homogeneous) quadratic form $Q$ and $k$ (homogeneous) linear forms $L_{1}, L_{2}, \ldots, L_{k}$, such that $\left.Q\right|_{\cap K e r} L_{i}$ is indefinite.

Assume that rank $Q \geq 2 k+3$. Then, for a residual, full measure set (in the induced topology/Lebesgue measure) of vectors $\mathbf{v} \neq \mathbf{0}$ in:

$$
\{Q=0\} \cap\left\{L_{i}=0,1 \leq i \leq k\right\}
$$

for any $\epsilon>0$, there are $\mathbf{x}_{n} \in \mathbb{Z}^{d}$, such that: 
(1) $\left\|\mathbf{x}_{n}\right\| \rightarrow \infty$,

(2) $\sup \left|Q\left(\mathbf{x}_{n}\right)\right|<\infty$,

(3) $\operatorname{dist}\left(\mathbf{x}_{n}, \mathbb{R}_{+} \mathbf{v}\right) \leq \epsilon$.

In particular,

$$
\left|L_{i}\left(\mathbf{x}_{n}\right)\right| \leq C \epsilon, \text { for all } 1 \leq i \leq k \text { and all } n
$$

with a constant $C>0$ determined by the linear forms.

There is a class of nilpotent groups, analogous to the Heisenberg groups, but with a compact center, for which we obtain stronger results and for which the proofs are much simpler.

The normal subgroup of $\mathcal{H}_{n}$ generated by $(0,0,1)$ is isomorphic to $\mathbb{Z}$. Denote by $\Gamma_{n}$ the quotient $\mathcal{H}_{n} / \mathbb{Z}$. The center of $\Gamma_{n}$ is $\mathbb{R} / \mathbb{Z} \cong S^{1}$; let $\widehat{\Gamma}_{n}=\Gamma_{n} / S^{1} \cong \mathbb{R}^{2 n}$.

If $\beta: X \rightarrow \Gamma_{n}$ is a cocycle, denote by $\widehat{\beta}: X \rightarrow \mathbb{R}^{2 n}$ the corresponding quotient cocycle. For $r>0$, let $\mathcal{S}^{r}\left(X, \Gamma_{n}\right)$ be the set of $C^{r}$ cocycles $\beta: X \rightarrow \Gamma_{n}$ for which $\widehat{\beta}$ is not cohomologous to a cocycle with values in a half-space.

Theorem 8 ([49, Theorem 1.5]). Assume that $X$ is a basic hyperbolic set for $f: X \rightarrow X$. Let $n \geq 1$, $r>0$. Then, there is an open and dense set of transitive cocycles in $\mathcal{S}^{r}\left(X, \Gamma_{n}\right)$.

\section{5. Г Is a Compact and Nilpotent Semidirect Product}

In this subsection, we follow [50].

Definition 7. A connected Lie group $\Gamma$ is called a good semidirect product if it is a semidirect product $K \ltimes N$, where $N$ is a nilpotent Lie group, $K$ is a compact Lie group and, in addition, if $T$ is a maximal torus in $K$; the only element of $N$ fixed under conjugation by $T$ is the identity.

Definition 8. A connected Lie group $\Gamma$ is perfect if its commutator subgroup $[\Gamma, \Gamma]$ coincides with $\Gamma$.

Theorem 9 ([50, Theorem 1.1]). Assume that $X$ is a hyperbolic basic set for $f: X \rightarrow X$. Let $\Gamma=K \ltimes N$ be a good semidirect product that is perfect. Then, in the class of $C^{r}$-cocycles $\beta: X \rightarrow \Gamma$, $r>0$, the transitive ones contain an open and dense set.

We describe next a large class of Lie groups that satisfy the assumptions in Theorem 9.

Definition 9. Let $\mathbb{K}$ be $\mathbb{R}$ or $\mathbb{C}$. Let $n$ be a positive integer, and let $n=n_{1}+n_{2}+\cdots+n_{\ell}$, where $n_{i}, 1 \leq i \leq \ell$ are also positive integers. Define $G\left(n_{1}, n_{2}, \ldots, n_{\ell}, \mathbb{K}\right)$ to be the subgroup of $G L(n, \mathbb{K})$, which consists of all block matrices:

$$
\left(\begin{array}{ccccc}
A_{1} & B_{1,2} & B_{1,3} & \ldots & B_{1, \ell} \\
0 & A_{2} & B_{2,3} & \ldots & B_{2, \ell} \\
0 & 0 & A_{3} & \ldots & B_{3, \ell} \\
\vdots & \vdots & \vdots & \ddots & \vdots \\
0 & 0 & 0 & \ldots & A_{\ell}
\end{array}\right)
$$


where:

- the block $A_{i}$ is an arbitrary matrix in the orthogonal group $S O\left(n_{i}\right)$ (respectively, the unitary group $\left.U\left(n_{i}\right)\right), 1 \leq i \leq \ell$;

- the block $B_{i, j}$ is an arbitrary matrix in $\operatorname{Mat}\left(n_{i}, n_{j}\right)$, for $1 \leq i \leq \ell-1, i+1 \leq j \leq \ell$,

- the blocks below the diagonal are zero.

Remark 5. For $n \geq 3$, the partition $(n-1,1)$ gives the special Euclidean group $S E(n-1)$.

Theorem 10 ([50, Theorem 1.2]). Let $\mathbb{K}$ be $\mathbb{R}$ or $\mathbb{C}$. Let $n \geq 3$ be an integer and $n_{1}+n_{2}+\cdots+n_{\ell}=n$ a partition of $n$, where $\left(n_{1}, n_{2}, \ldots, n_{\ell}\right)$ are even integers, all greater or equal to four, except possibly for a single occurrence of one. Then, $\Gamma=G\left(n_{1}, n_{2}, \ldots, n_{\ell}, \mathbb{K}\right)$ is a good semidirect product, which is perfect.

Problem 3. If $n_{i}=2$, for some $1 \leq i \leq \ell$, and if there is no more than one one among the $n_{i}$ 's, then the group $G\left(n_{1}, n_{2}, \ldots, n_{\ell}, \mathbb{R}\right)$ is a good semidirect product that is not perfect. If there are at least two ones among the $n_{i}$ 's, then the group $G\left(n_{1}, n_{2}, \ldots, n_{\ell}, \mathbb{R}\right)$ is not a good semidirect product. With the exception of the case $G=S E(2)=G(2,1, \mathbb{R})$, solved in [46] using results about extensions with Abelian fiber, Theorem 10 leaves open the transitivity conjecture for these cases.

Definition 10. If $\Gamma$ is a topological group, we call $g \in \Gamma$ compact if the closure of the subgroup generated by $g$ is compact. We denote by $\mathcal{C}(\Gamma)$ the set of compact elements in $\Gamma$.

Remark 6. The proof of Theorem 9 is based on the existence of an open dense set of compact elements in $\Gamma$. It is shown in [51] that if a connected Lie subgroup $H$ of $G L(n, \mathbb{K})$ contains an open neighborhood of the identity in which compact elements are dense and is maximal with this property, then $H$ is conjugate to a group $G\left(n_{1}, n_{2}, \ldots, n_{\ell}, \mathbb{K}\right)$. This remark shows the optimality of our results and the limitations of the method. In order to solve Problem 3, one needs to develop new techniques.

\section{6. Г Is a Non-Compact Semisimple Lie Group}

The conjecture is not verified for any non-compact semisimple Lie group. Nevertheless, for $\Gamma=S L(2, \mathbb{R})$ and, more generally, for $\Gamma=S p(n, \mathbb{R})$, open sets of transitive extensions are constructed in [33]. Here is a brief description of the construction. One uses the existence of compact elements in $\Gamma$ that are stably compact under small perturbations. No such elements are known in $S L(3, \mathbb{R})$ or many other non-compact semisimple Lie groups. These compact elements can be chosen arbitrarily close to the identity. We start with the identity cocycle $\beta$. After a small perturbation, we can arrange for the height of $\beta$ over the orbit of a fixed periodic point $x_{0} \in X$ to be a stably compact element. Using the method described in Section 4, this allows one to construct a set of generators for $\Gamma$ in the range $\mathcal{L}_{\beta}\left(x_{0}\right)$ (defined in Section 4) that are close to the identity. The generating property is stable under small perturbations due to a classical result of Kuranishi [52]. In conjunction with Theorem 11 in Section 4, this gives a stably transitive extension with fiber $S p(n, \mathbb{R})$.

The following problem is left open: 
Problem 4. For $\alpha \in(0,1)$, find a $C^{\alpha}$-stable transitive $S L(3, \mathbb{R})$-extension of an Anosov diffeomorphism.

\section{Criterion for Transitivity}

In order to prove the topological transitivity of extensions with non-compact fiber, sometimes we are able to use the methods developed by Brin in the proof of his general result mentioned in the Introduction. This is due to the existence of the pair of stable/unstable foliations for the extension and the fact that generic accessibility for the pair is easy to prove in some cases. The difficult part is to show the density of recurrent points. The compact factors in $\Gamma$ are sometimes easy to accommodate, due to the existence of compact elements. See [46] for the case of $\Gamma=S E(2)$ and $\Gamma=K \times \mathbb{R}^{n}$ with $K$ compact.

We denote by $W^{s}(x)$ and $W^{u}(x)$ the stable and unstable leaves of the hyperbolic dynamical system $f$ through the point $x \in X$. The next lemma is a consequence of [20, Appendix A]. Detailed proofs can be found in [53].

Lemma 1. Assume that $X$ is a hyperbolic basic set for $f: X \rightarrow X$, that $\Gamma$ is a connected Lie group and $\beta: X \rightarrow \Gamma$ an $\alpha$-Hölder cocycle that has subexponential growth. Then, the $\Gamma$-extension $f_{\beta}: X \times \Gamma \rightarrow$ $X \times \Gamma$ admits stable and unstable foliations, which are $\alpha$-Hölder and invariant under right multiplication by elements of $\Gamma$. The stable and unstable leaves of $f_{\beta}$ through $\left(x, e_{\Gamma}\right) \in X \times \Gamma$ are the graphs of the functions:

$$
\begin{aligned}
& \gamma_{x}^{s}: W^{s}(x) \rightarrow \Gamma, \quad \gamma_{x}^{s}(y)=\lim _{n \rightarrow \infty} \beta(n, x) \beta(n, y)^{-1} \\
& \gamma_{x}^{u}: W^{u}(x) \rightarrow \Gamma, \quad \gamma_{x}^{u}(y)=\lim _{n \rightarrow \infty} \beta(-n, x) \beta(-n, y)^{-1}
\end{aligned}
$$

These functions are $\alpha$-Hölder and vary continuously with the cocycle $\beta$ in the following sense: if $\beta_{k} \rightarrow \beta$ in the $C^{0}$-topology and $\beta_{k}$ remains $C^{\alpha}$-bounded, then $\gamma_{k, x}^{s} \rightarrow \gamma_{x}^{s}$ and $\gamma_{k, x}^{u} \rightarrow \gamma_{x}^{u}$ on $W_{l o c}^{s}(x)$ in the $C^{0}$-topology.

We call the values of the functions $\gamma_{x}^{s}, \gamma_{x}^{u}$ holonomies along stable/unstable leaves.

A new criterion for topological transitivity applicable to extensions was developed in [33]. One of the key notions introduced in [33] is:

Definition 11. Let $\Gamma$ be a connected Lie group, $X$ a basic hyperbolic set for $f: X \rightarrow X, \beta: X \rightarrow \Gamma a$ cocycle and $f_{\beta}: X \times \Gamma \rightarrow X \times \Gamma$ the skew-extension. Given $x \in X$, let:

$$
\mathcal{L}_{\beta}(x)=\left\{\gamma \in \Gamma: \text { there exist } x_{k} \in X \text { and } n_{k}>0 \text { such that } x_{k} \rightarrow x \text { and } f_{\beta}^{n_{k}}\left(x_{k}, e_{\Gamma}\right) \rightarrow(x, \gamma)\right\}
$$

We will refer to $\mathcal{L}_{\beta}(x)$ as the range of $\beta$ over $x$.

That is, the set $\mathcal{L}_{\beta}(x)$ consists of the possible limits $\lim _{k \rightarrow \infty} \beta\left(n_{k}, x_{k}\right)$, subject to $x_{k} \rightarrow x$ and $f^{n_{k}}\left(x_{k}\right) \rightarrow x$. Note that we do not require that $n_{k} \rightarrow \infty$ or that $x_{k} \neq x$. Clearly, $\mathcal{L}_{\beta}(x)$ is a closed subset of $\Gamma$.

The following theorem is [33, Lemma 3.1, Theorem 3.3].

Theorem 11. Assume that $X$ is a hyperbolic basic set for $f: X \rightarrow X$, that $\Gamma$ is a connected Lie group and $\beta: X \rightarrow \Gamma$ a Hölder cocycle that has subexponential growth. Then: 
(1) $\mathcal{L}_{\beta}(x)$ is a closed semigroup of $\Gamma$ for each $x \in X$.

(2) If there exists a point $x_{0} \in X$, such that $\mathcal{L}_{\beta}\left(x_{0}\right)=\Gamma$, then $\beta$ is a transitive cocycle.

The following lemma is [33, Lemma 2.2]. Briefly, it says that the heights of $f_{\beta}$ over two nearby trajectories in the base are close, independent of the lengths of the trajectories. This result is used in the constructions of elements in $\mathcal{L}_{\beta}$.

Lemma 2. Assume that $X$ is a hyperbolic basic set for $f: X \rightarrow X$, that $\Gamma$ is a connected Lie group and $\beta: X \rightarrow \Gamma$ an $\alpha$-Hölder cocycle that has subexponential growth. Then, there is a constant $C_{5}>0$ with the following property.

For any $\epsilon>0$ sufficiently small, any $n \geq 1$ and any two trajectories $x_{k}=f^{k}\left(x_{0}\right), y_{k}=f^{k}\left(y_{0}\right)$, such that $d_{M}\left(x_{k}, y_{k}\right)<\epsilon$ for $0 \leq k \leq n-1$,

$$
d\left(\beta\left(n, x_{0}\right), \beta\left(n, y_{0}\right)\right) \leq C_{5}\left(\left\|\operatorname{Ad}\left(\beta\left(n, x_{0}\right)\right)\right\|+1\right) \epsilon^{\alpha}
$$

\section{Admissible Sequences of Products of Holonomies}

We observe that a priori, the set $\mathcal{L}_{\beta}$ introduced in the previous section may be empty. In this section, we describe a method for obtaining elements in $\mathcal{L}_{\beta}$. We follow closely [47].

Throughout this section, $\left(M, d_{M}\right)$ is a Riemannian manifold, $X \subset M$ is a basic hyperbolic set for $f: X \rightarrow X$ with contraction constant $\lambda \in(0,1)$ satisfying (1), $\Gamma$ a connected Lie group and $\beta: X \rightarrow \Gamma$ an $\alpha$-Hölder cocycle that has subexponential growth.

Definition 12. By a periodic heteroclinic cycle, we mean a cycle consisting of points $p_{1}, \ldots, p_{k}$ that are periodic for the map $f$, have disjoint trajectories, such that $p_{j}$ is transverse heteroclinic to $p_{j+1}$ through a point $\zeta_{j} \in W^{u}\left(p_{j}\right) \cap W^{s}\left(p_{j+1}\right)$, for $j=1, \ldots, k$ (where $\left.p_{k+1}=p_{1}\right)$.

Let $P_{1}, \ldots, P_{k}$ be the corresponding periodic orbits and denote the periods by $\ell_{1}, \ldots, \ell_{k}$. Denote by $O_{j}$ the heteroclinic trajectory from $p_{j}$ to $p_{j+1}$ (of the point $\zeta_{j}$ chosen above), and by $H_{j}$ the holonomy along this heteroclinic connection (that is, along $W^{u}\left(p_{j}\right)$ from $p_{j}$ to $\zeta_{j}$ and then along $W^{s}\left(p_{j+1}\right)$ from $\zeta_{j}$ to $\left.p_{j+1}\right)$.

Replace the heteroclinic orbit $O_{j}$ from $p_{j}$ to $p_{j+1}$ by the trajectory $Q_{j}$ of length $\ell_{j} M_{j}+\ell_{j+1} M_{j+1}$ that spends time $\ell_{j} M_{j}$ in the first half of $O_{j}$ and time $\ell_{j+1} M_{j+1}$ in the second half of $O_{j}$; that is, $Q_{j}=\left\{f^{n}\left(\zeta_{j}\right) \mid-\ell_{j} M_{j} \leq n<0\right\} \cup\left\{f^{n}\left(\zeta_{j}\right) \mid 0 \leq n<\ell_{j+1} M_{j+1}\right\}$ ). For the trajectory connecting $p_{k}$ to $p_{k+1}$, we allow $M_{1}$ and $M_{k+1}$ to be distinct. The positive integers $M_{j}$ will be chosen later.

Consider the heights $\beta\left(P_{j}\right)$ and $\beta\left(Q_{j}\right)$ over the periodic orbits $P_{j}$ and trajectories $Q_{j}$.

Lemma 3. For $j=1, \ldots, k$, the limit:

$$
\lim _{M_{j}, M_{j+1} \rightarrow \infty} \beta\left(P_{j}\right)^{-M_{j}} \beta\left(Q_{j}\right) \beta\left(P_{j+1}\right)^{-M_{j+1}}=H_{j}
$$

exists and is the product of the holonomies along the unstable and stable leaves of $O_{j}$, from $p_{j}$ to $p_{j+1}$.

Proof. This follows from Lemma 1. 
Definition 13. Consider a sequence of vectors $N(1), N(2) \ldots \in \mathbb{N}^{k+1}$ whose entries are positive integers. Write $N(i)=\left(M_{1}(i), \ldots, M_{k+1}(i)\right)$. The sequence is admissible if there is a constant $C_{2} \geq 1$, such that $M_{p}(i) / M_{q}(i) \leq C_{2}$, for all $p, q=1 \ldots, k+1$ and all $i \geq 1$.

If $N=\left(M_{1}, \ldots, M_{k+1}\right)$ is a sequence, we write $N \rightarrow \infty$ if $M_{p} \rightarrow \infty$ for $p=1, \ldots, k+1$.

Theorem 12. Let $N=\left(M_{1}, \ldots, M_{k+1}\right) \in \mathbb{N}^{k+1}$. Define:

$$
A(N)=\beta\left(P_{1}\right)^{M_{1}} H_{1} \beta\left(P_{2}\right)^{2 M_{2}} H_{2} \cdots \beta\left(P_{k}\right)^{2 M_{k}} H_{k} \beta\left(P_{1}\right)^{M_{k+1}}
$$

If the limit $A=\lim _{N \rightarrow \infty} A(N)$ exists along an admissible sequence $N(1), N(2), \ldots$, then $A \in \mathcal{L}_{\beta}\left(p_{1}\right)$.

In the remainder of this section, we prove Theorem 12. From now on, we assume for notational simplicity that $P_{j}=p_{j}$ are fixed points (so, $\ell_{j}=1$ ).

Given $N=\left(M_{1}, \ldots, M_{k+1}\right) \in \mathbb{N}^{k+1}$, define:

$$
|N|=\left(M_{1}+M_{k+1}\right) / 2+\sum_{j=2}^{k} M_{j}, \min N=\min \left\{M_{1}, \ldots, M_{k+1}\right\}, \max N=\max \left\{M_{1}, \ldots, M_{k+1}\right\}
$$

Note that for an admissible sequence $N$, we have $\max N \leq C_{2} \min N$. Define:

$$
H_{j}(N)=\beta\left(P_{j}\right)^{-M_{j}} \beta\left(Q_{j}\right) \beta\left(P_{j+1}\right)^{-M_{j+1}} .
$$

By Lemma 3, $\lim _{N \rightarrow \infty} H_{j}(N)=H_{j}$ (independent of the sequence $N$ ). Moreover, by [20, proof of Theorem 4.3(g)], there is $\delta_{0} \in(0,1)$, such that:

$$
d\left(H_{j}(N), H_{j}\right)=O\left(\delta_{0}^{\min N}\right)
$$

Recall that $Q_{j}$ is a trajectory of length $M_{j}+M_{j+1}$ that shadows the heteroclinic connection from $p_{j}$ to $p_{j+1}$. Concatenate these trajectories to form a periodic pseudo-orbit $Q=Q_{1} \ldots Q_{k}$ of length $2|N|$. Then, $Q$ is a $\delta$-pseudo-orbit with $\delta \leq C_{3} \lambda^{\min N}$, where $C_{3}>0$ is a constant (depending on $f: X \rightarrow X$ ) and $\lambda$ is the contraction constant. By the hyperbolicity of $X$, there is a periodic orbit $\tilde{Q}$ of length $2|N|$ that $\epsilon$-shadows $Q$ with $\epsilon \leq C_{4} \lambda^{\min N}$, where $C_{4}>0$ is a constant. See [54, page 74] for standard shadowing techniques.

\section{Proposition 1.}

(1) $\beta(Q)=\beta\left(P_{1}\right)^{M_{1}} H_{1}(N) \beta\left(P_{2}\right)^{2 M_{2}} H_{2}(N) \cdots \beta\left(P_{k}\right)^{2 M_{k}} H_{k}(N) \beta\left(P_{1}\right)^{M_{k+1}}$.

(2) $\lim _{N \rightarrow \infty} d(\beta(Q), \beta(\tilde{Q}))=0$ along admissible sequences $N$.

(3) $\lim _{N \rightarrow \infty} d(\beta(Q), A(N))=0$ along admissible sequences $N$.

Proof. Part (1) is a direct calculation, namely:

$$
\beta(Q)=\prod_{j=1}^{k} \beta\left(Q_{j}\right)=\prod_{j=1}^{k} \beta\left(P_{j}\right)^{M_{j}} H_{j}(N) \beta\left(P_{j+1}\right)^{M_{j+1}}
$$


Next, write $\tilde{Q}=\tilde{Q}_{1} \ldots \tilde{Q}_{k}$ where $\tilde{Q}_{j}$ has length $M_{j}+M_{j+1}$. Define $\gamma_{j}=\beta\left(Q_{j}\right), \tilde{\gamma}_{j}=\beta\left(\tilde{Q}_{j}\right)$. Note that $Q_{j}$ and $\tilde{Q}_{j}$ have a length of at most $2 \max N$ and that $\tilde{Q}_{j} \epsilon$-shadows $Q_{j}$ with $\epsilon \leq C_{4} \lambda^{\min N}$. It follows from Lemma 2 that $d\left(\gamma_{i}, \tilde{\gamma}_{i}\right) \leq C \lambda^{\alpha \min N}\left(\left\|\operatorname{Ad}\left(\gamma_{i}\right)\right\|+1\right)$ where $C=C_{4}^{\alpha} C_{5}$. Hence, using the properties of the metric on $\Gamma$ and the fact that $\beta$ has subexponential growth, we have:

$$
\begin{aligned}
d(\beta(Q), \beta(\tilde{Q}))= & d\left(\gamma_{1} \gamma_{2} \cdots \gamma_{k}, \tilde{\gamma}_{1} \tilde{\gamma}_{2} \cdots \tilde{\gamma}_{k}\right) \\
\leq & d\left(\gamma_{1} \gamma_{2} \cdots \gamma_{k}, \tilde{\gamma}_{1} \gamma_{2} \cdots \gamma_{k}\right)+d\left(\tilde{\gamma}_{1} \gamma_{2} \gamma_{3} \cdots \gamma_{k}, \tilde{\gamma}_{1} \tilde{\gamma}_{2} \gamma_{3} \cdots \gamma_{k}\right)+ \\
& \quad \cdots+d\left(\tilde{\gamma}_{1} \tilde{\gamma}_{2} \cdots \tilde{\gamma}_{k-1} \gamma_{k}, \tilde{\gamma}_{1} \tilde{\gamma}_{2} \cdots \tilde{\gamma}_{k-1} \tilde{\gamma}_{k}\right) \\
\leq & d\left(\gamma_{1}, \tilde{\gamma}_{1}\right)\left\|\operatorname{Ad}\left(\gamma_{2} \cdots \gamma_{k}\right)\right\|+d\left(\gamma_{2}, \tilde{\gamma}_{2}\right)\left\|\operatorname{Ad}\left(\gamma_{3} \cdots \gamma_{k}\right)\right\|+\cdots+d\left(\gamma_{k}, \tilde{\gamma}_{k}\right) \\
\leq & C \lambda^{\alpha \min N}\left[(1+\eta)^{2 \max N}+1\right]\left[(1+\eta)^{2 \max N}+\cdots+(1+\eta)^{2(k-1) \max N}\right]
\end{aligned}
$$

where $\eta>0$ can be chosen arbitrarily small and $\|\operatorname{Ad}(\beta(n, x))\| \leq(1+\eta)^{n}$ for $n$ large enough. Restricting to admissible sequences, $\min N$ and $\max N$ are comparable, and Part (2) follows. The proof of Part (3) is similar using (5).

Proof of Theorem 12. By assumption, $A(N) \rightarrow A$. Hence, by Proposition 1, Part (2,3), $\beta(\tilde{Q}) \rightarrow A$. We conclude that $A \in \mathcal{L}_{\beta}\left(p_{1}\right)$ by definition of $\mathcal{L}_{\beta}\left(p_{1}\right)$.

Remark 7. Proving that the limit $A=\lim _{N \rightarrow \infty} A(N)$ exists is challenging. The proof depends on the group that appears in the fiber. If $\Gamma$ is the Heisenberg group, the components of $A(N)$ are a quadratic and several linear polynomials. In order to extract a convergent subsequence, we need to show that a diophantine system of a quadratic and several linear equations has approximate solutions. A difficulty is that, even though we can prescribe the leading coefficients in these equations, we cannot control all of the coefficients. Lower order coefficients depend on the holonomy factors in $A(N)$, which are difficult to control generically. This is the place where the diophantine approximation results, such as Theorem 7 , come into play.

\section{Semigroup Problem}

For many Lie groups $\Gamma$, it is not hard to show that, for an integer $p>0$ big enough, there is a large open set $U \subset \Gamma^{p}$, such that if $F \in U$, then the family $F$ generates $\Gamma$, that is the group generated by $F$ is dense in $\Gamma$. The proof of transitivity of an extension $f_{\beta}$ is based on showing that the set $\mathcal{L}_{\beta}(x)$ of "heights" of $\beta$ over a (periodic) point $x$ is the whole fiber $\Gamma$. See Theorem 11. To obtain the condition $\mathcal{L}_{\beta}(x)=\Gamma$, we have to prove that for a typical family $F \in \Gamma^{p}$ that generates $\Gamma$ as a group, if $F$ is not contained in a maximal sub-semigroup with a non-empty interior, then $F$ generates $\Gamma$ as a semigroup, as well. We refer to this question as the semigroup problem. For example, if $\Gamma=\mathbb{R}$, the problem states that if a set $S$ contains both positive and negative numbers, then the closure of the semigroup generated by $S$ is a group.

The semigroup problem was solved for $\Gamma=\mathbb{R}^{n}$ [41] and more generally for groups of the form $\Gamma=K \times \mathbb{R}^{n}$, where $K$ is a compact Lie group [33, Theorem 5.10]. It is also solved for $\Gamma=S E(n)$ [33, Theorem 6.8], for certain solvable groups that are semidirect products of $\mathbb{R}^{n}$ with $\mathbb{R}^{m}$, such as $\mathrm{Aff}^{+}$in [55], and for the Heisenberg group in [48]. 


\section{Acknowledgments}

Viorel Nitica was partially supported by the Simons Foundation Grant 208729. Andrew Török was partially supported by the Simons Foundation Grant 239583.

\section{Conflicts of Interest}

The authors declare no conflict of interest.

\section{References}

1. Blanchard, F. Topological chaos: What may this mean? J. Differ. Equ. Appl. 2009, 15, 23-46.

2. Kolyada, S.; Snoha, L. Some aspects of topological transitivity-A survey. Grazer Math. Ber. 1997, 334, 3-35.

3. Devaney, R.L. An Introduction to Chaotic Dynamical Systems, 2nd ed.; Addison-Wesley: Redwood City, CA, USA, 1989.

4. Banks, J.; Brooks, J.; Cairns, J.; Davis, G.; Stacey, P. On Devaney's definition of chaos. Am. Math. Monthly 1992, 99, 332-334.

5. Oxtoby, J.; Ulam, S. Measure-preserving homeomorphisms and metrical transitivity. Ann. Math. 1941, 42, 874-920.

6. Besicovitch, A.S. A problem on topological transformation of the plane. Fund. Math. 1937, 28, $61-65$.

7. Besicovitch, A.S. A problem on topological transformation of the plane II. Proc. Cambr. Phil. Soc. 1951, 47, 38-45.

8. Shnirelman, L.G. An example of a transformation of the plane. Izv. Donsk. Polytech. Inst. 1930, 14, 64-74. (In Russian)

9. Sidorov, Ye. A. Topologically transitive cylindrical cascades. Mat. Zametki 1973, 14, 441-452. (In Russian)

10. Anosov, D. V.; Sinai, Ja.G. Certain smooth ergodic systems. Uspehi Mat. Nauk 1967, 22, 107-172. (In Russian)

11. Bowen, R. Equilibrium States and the Ergodic Theory of Anosov Diffeomorphisms; Lecture Notes in Mathematics; Springer-Verlag: Berlin, Germany, 1975; Volume 470.

12. Smale, S. Differentiable dynamical systems. Bull. Amer. Math. Soc. 1967, 73, 747-817.

13. Katok, A.; Hasselblatt, B. Introduction to the Modern Theory of Dynamical Systems; Cambridge University Press: Cambridge, UK, 1995.

14. Hopf, E. Statistik der geodätischen Linien in Mannigfaltigkeiten negativer Krümmung. Ber. Verh. Sachs. Akad. Wiss. Leipzig 1939, 91, 261-304. (In German)

15. Anosov, D.V. Geodesic Flows on Closed Riemannian Manifolds with Negative Curvature. Trudy Mat. Inst. Steklov 1967, 90, 209. (In Russian)

16. Brin, M.; Pesin, Y. Partially hyperbolic dynamical systems. Izvestia Akad. Nauk SSSR Ser. Mat. 1974, 38, 170-212. (In Russian)

17. Grayson, M.; Pugh, C.; Shub, M. Stably ergodic diffeomorphisms. Ann. of Math. 1994, 140, 295-329. 
18. Pugh, C.; Shub, M. Stable ergodicity and julienne quasi-conformality. J. Eur. Math. Soc. 2000, 2, $1-52$.

19. Burns, K.; Wilkinson, A. On the ergodicity of partially hyperbolic systems. Ann. of Math. 2010, 171, 451-489.

20. Nitica, V.; Török, A. An open and dense set of stably ergodic diffeomorphisms in a neighborhood of certain nonergodic ones. Topology 2001, 40, 259-278.

21. Hertz, F. R. Stable ergodicity of certain linear automorphisms of the torus. Ann. Math. 2005, 162, 65-107.

22. Dolgopyat, D; Wilkinson, A. Stable accessibility is $C^{1}$ dense. In Geometric Methods in Dynamics II, 2003; Volume 287, pp. 33-60.

23. Brin, I.M. Topological transitivity of a class of dynamical systems and frame flow on manifolds of negative curvature. Funct. Anal. Appl. 1975, 9, 9-19.

24. Bonatti, C.; Díaz, L.J. Persistent nonhyperbolic transitive diffeomorphisms. Ann. of Math. 1996, 143, 357-396.

25. Shub, M. Topological transitive diffeomorphism on $T^{4}$. Lect. Notes in Math. 1971, 206, 39-40.

26. Bonatti, C.; Crovisier, S. Récurrence et généricité. Invent. Math. 2004, 158, 33-104. (In French)

27. Pugh, C. The closing lemma. Amer. J. Math. 1967, 89, 956-1009.

28. Hayashi, S. Connecting invariant manifolds and the solution of the $C^{1}$-stability and $Q$-stability conjectures for flows. Ann. Math. 1997, 145, 81-137.

29. Hayashi, S. Corrections to: "Connecting invariant manifolds and the solution of the $C^{1}$-stability and $Q$-stability conjectures for flows". Ann. Math. 1999, 150, 353-356.

30. Grosse-Erdmann, K.-G.; Peris, A. Linear Chaos; Springer: Berlin, Germany, 2011.

31. Pollicott, M.; Walkden, C.P. Livšic theorems for connected Lie groups. Trans. Amer. Math. Soc. 2001, 353, 2879-2895.

32. Dungey, N.; ter Elst, A.F.M.; Robinson, D.W. Analysis on Lie Groups with Polynomial Growth; Progress in Mathematics; Birkhauser Boston Inc.: Boston, MA, USA, 2003.

33. Melbourne, I.; Nitica, V.; Török, A. Stable transitivity of certain noncompact extensions of hyperbolic systems. Ann. Henri Poincare 2005, 6, 725-746.

34. Nitica, V. A note about topologically transitive cylindrical cascades. Isr. J. Math. 2001, 126, 141-156.

35. Nitica, V. Examples of topologically transitive skew-products. Discret. Contin. Dyn. Syst. 2000, 6, 351-360.

36. Field, M.; Melbourne, I.; Török, A. Stable ergodicity for smooth compact Lie group extensions of hyperbolic basic sets. Ergodic Theory Dyn. Syst. 2005, 25, 517-551.

37. Field, M.; Nitica, V. Stable topological transitivity of skew and principal extensions. Nonlinearity 2001, 14, 1055-1069.

38. Field, M.; Parry, W. Stable ergodicity of skew extensions by compact Lie groups. Topology 1999, 167-187.

39. Parry, W.; Pollicott, M. Stability of mixing for toral extensions of hyperbolic systems. Proc. Steklov Inst. Math. 1990, 216, 350-359. 
40. Burns, K.; Wilkinson, A. Stable ergodicity of skew products. Ann. Sci. Ecole Norm. Sup. 1999, 32, 859-889.

41. Nitica, V.; Pollicott, M. Transitivity of Euclidean extensions of Anosov diffeomorphisms. Ergod. Theory Dyn. Syst. 2005, 25, 257-269.

42. Bousch, T. La condition de Walter. Ann. Sci. Ecole Norm. Sup. 2001, 34, 287-311. (In French)

43. Bowen, R. On Axiom A Diffeomorphisms; American Mathematical Society: Providence, RI, USA, 1977; Volume 35.

44. Moss, A.; Walkden, C.P. Stable topological transitivity properties of $\mathbb{R}^{n}$-extensions of hyperbolic transformations. Ergod. Theory Dyn. Syst. 2012, 32, 1435-1443.

45. Melbourne, I.; Nicol, M. Stable transitivity of Euclidean group extensions. Ergod. Theory Dyn. Syst. 2003, 23, 611-619.

46. Melbourne, I.; Nitica, V.; Török, A. A note about stable transitivity of noncompact extensions of hyperbolic systems. Discret. Contin. Dyn. Syst. 2006, 14, 355-363.

47. Melbourne, I.; Nitica, V.; Török, A. Transitivity of Euclidean-type extensions of hyperbolic systems. Ergod. Theory Dyn. Syst. 2009, 29, 1585-1602.

48. Nitica, V.; Török, A. Stable transitivity of Heisenberg group extensions of hyperbolic systems. Nonlinearity 2014, 27, 661-683.

49. Melbourne, I.; Nitica, V.; Török, A. Transitivity of Heisenberg group extensions of hyperbolic systems. Ergod. Theory Dyn. Syst. 2012, 32, 223-235.

50. Nitica, V. Stably transitivity for extensions of hyperbolic systems by semidirect products of compact and nilpotent Lie groups. Discret. Contin. Dyn. Syst. 2011, 29, 1197-1203.

51. Djoković, D.Z. The union of compact subgroups of a connected locally compact group. Math. Z. 1978, 158, 99-105.

52. Kuranishi, M. On everywhere dense imbeddings of free groups in Lie groups. Nagoya Math. J. 1951, 2, 63-71.

53. Katok, A.; Nitica, V. Rigidity of Abelian Group Actions. I, Introduction to Cocycle Problem; Cambridge University Press: Cambridge , UK, 2011.

54. Newhouse, S. Lectures on Dynamical Systems. In Dynamical Systems; Progress in Mathematics; Springer: Berlin, Germany, 1980; pp. 1-114.

55. Lui, K.; Nitica, V.; Venkatesh, S. The semigroup problem for central semidirect product of $\mathbb{R}^{n}$ with $\mathbb{R}^{m}$. Topol. Proc. 2015, 45, 9-29.

(c) 2015 by the authors; licensee MDPI, Basel, Switzerland. This article is an open access article distributed under the terms and conditions of the Creative Commons Attribution license (http://creativecommons.org/licenses/by/4.0/). 